\begin{tabular}{ll}
\hline \hline MINING AND METALLURGY INSTITUTE BOR & ISSN: 2334-8836 (Štampano izdanje) \\
UDK: 622 & ISSN: 2406-1395 (Online) \\
\hline
\end{tabular}

Branislav Rajković, Bojan Drobnjaković, Milenko Jovanović, Marko Mitrović*

\title{
DIMENSIONING OF AIR PIPELINE FOR THE SECONDARY CRUSHER DEDUSTING IN THE FLOTATION PLANT BOR
}

\begin{abstract}
This work gives the calculation methodology of air pipeline for dusty air removal on the example of air pipeline for the HP 200 secondary crusher dedusting, designed for the needs of reconstruction the Flotation Plant Bor. The analysis was done by calculation and includes checking the loss of pressure in the pipeline, taking into account the presence of dust. Graphical representation of the pipeline is also given, as well as its characteristic elements.
\end{abstract}

Keywords: air pipeline for dusty air removal, calculation, pressure loss in the pipeline

\section{INTRODUCTION}

Project of reconstruction the Flotation Plant Bor [1] has also included the Old Flotation Crushing Plant in terms of assembly the new equipment for crushing, screening and transport of slag from the New Smelter in Bor. In order to keep working environment conditions within the norms prescribed by the by standard SRPS Z.B0.001 [2], a dedusting system was designed [3]. The dedusting system provides that the dust emission to the atmosphere after purification to be below the limit value of emission the noxious and dangerous matters into the air at the place of pollution source determined by corresponding regulations [4]. The dedusting system was designed as an installation for dry dust extraction with forced air circulation where dusty air is removed from the place of pollution sources (ten suction places), purified in a bag house pulse jet filter (label FKK-750.s, manufacturer Termometal Belgrade) and discharged into atmosphere as purified air. Forced air circulation is provided by a centrifugal medium pressure fan (label R5A.100.10, manufacturer Termometal Belgrade) placed downstream of the bag house pulse jet filter.

\section{TECHNICAL DESCRIPTION}

In order to prevent dust emission from the HP 200 secondary crusher, it is covered from the top by a hood with aperture for material loading into the crusher and aperture for air pipeline connection for dusty air removal from this suction place. Dusty air is transported from the secondary crusher by the air pipeline $\varnothing 315$ to the collecting air pipeline $\varnothing 1000$ in front of the bag house pulse jet filter.

Air pipeline for the secondary crusher dedusting consists of straight segments and fittings with outer diameter of $\mathrm{D}=315 \mathrm{~mm}$. Elements of the pipeline are made by welding from sheet steel S235JRG2 (Č.0361) with thickness of $3 \mathrm{~mm}$. The pipeline segments are mutually joined by flanges with thickness of $5 \mathrm{~mm}$, whereby a simple

\footnotetext{
* Mining and Metallurgy Institute Bor, branislav.rajkovic@irmbor.co.rs
} 
assembly and disassembly of pipeline elements is enabled. The pipeline is connected to the suction hood through an elastic element preventing the vibrations of the secondary crusher to expand to the pipeline. A manually actuated round control damper is placed above this connection point for pipe network balancing. The pipeline bends are made as segmented bends with radius of $r=., 5 \mathrm{D}$ reinforc ed on the outer side of the curve. The branch at the connection point of this pipeline to the collecting air pipeline $\varnothing 1000$ has connection angle of $30^{\circ}$ in relation to the axis of the collecting air pipeline. The pipeline runs along the existing steel structure in the hall of the Old Flotation Crushing Plant being suitably supported on it, on the outer side, the pipeline goes along the wall of the building and then is connected to the collecting air pipeline. The pipeline is primed protected and coated by final paint.

Pipeline view in the cross section of the hall is given in Figure 1.

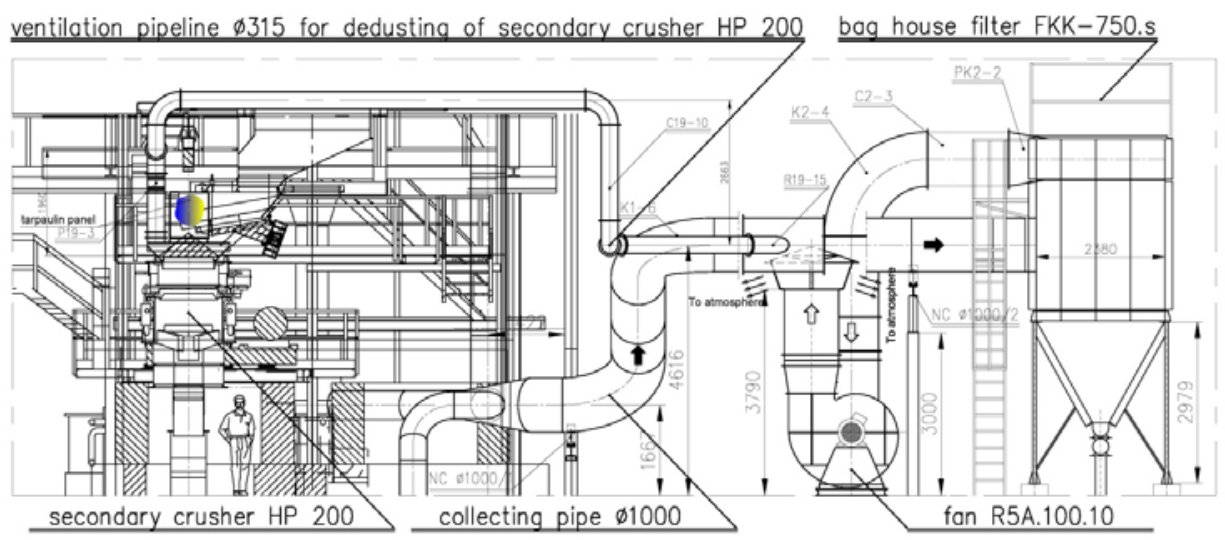

Figure 1 Pipeline for the HP 200 secondary crusher dedusting in the cross section of the hall of the Old Flotation Crushing Plant

The pipeline view for the HP 200 secondary crusher dedusting and part of the collecting air pipeline between the building and wall in front of the bag filter is given in Figure 2 in isometric view.

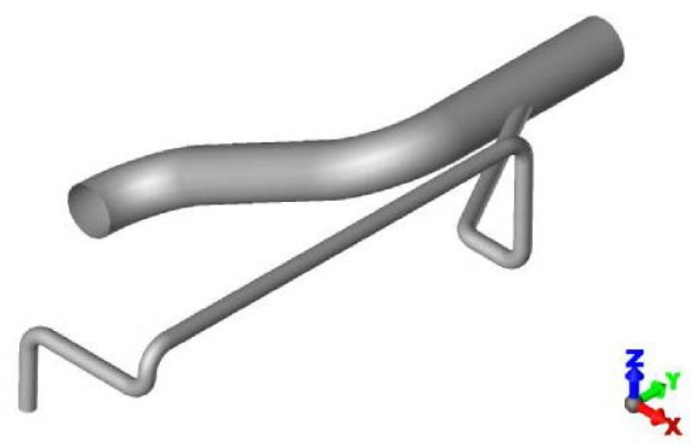

Figure 2 Isometric view of pipeline for the secondary crusher dedusting $\varnothing 315$ and segment of the collecting pipeline $\varnothing 1000$ 
Fittings of the pipeline are segmented the collecting pipeline $\varnothing 1000$. These fibends of $90^{\circ}$ and $60^{\circ}$, as well as the branch ttings are shown in Figure 3, as well as a at the connection point of pipeline $\varnothing 315$ to typical support and round control damper.
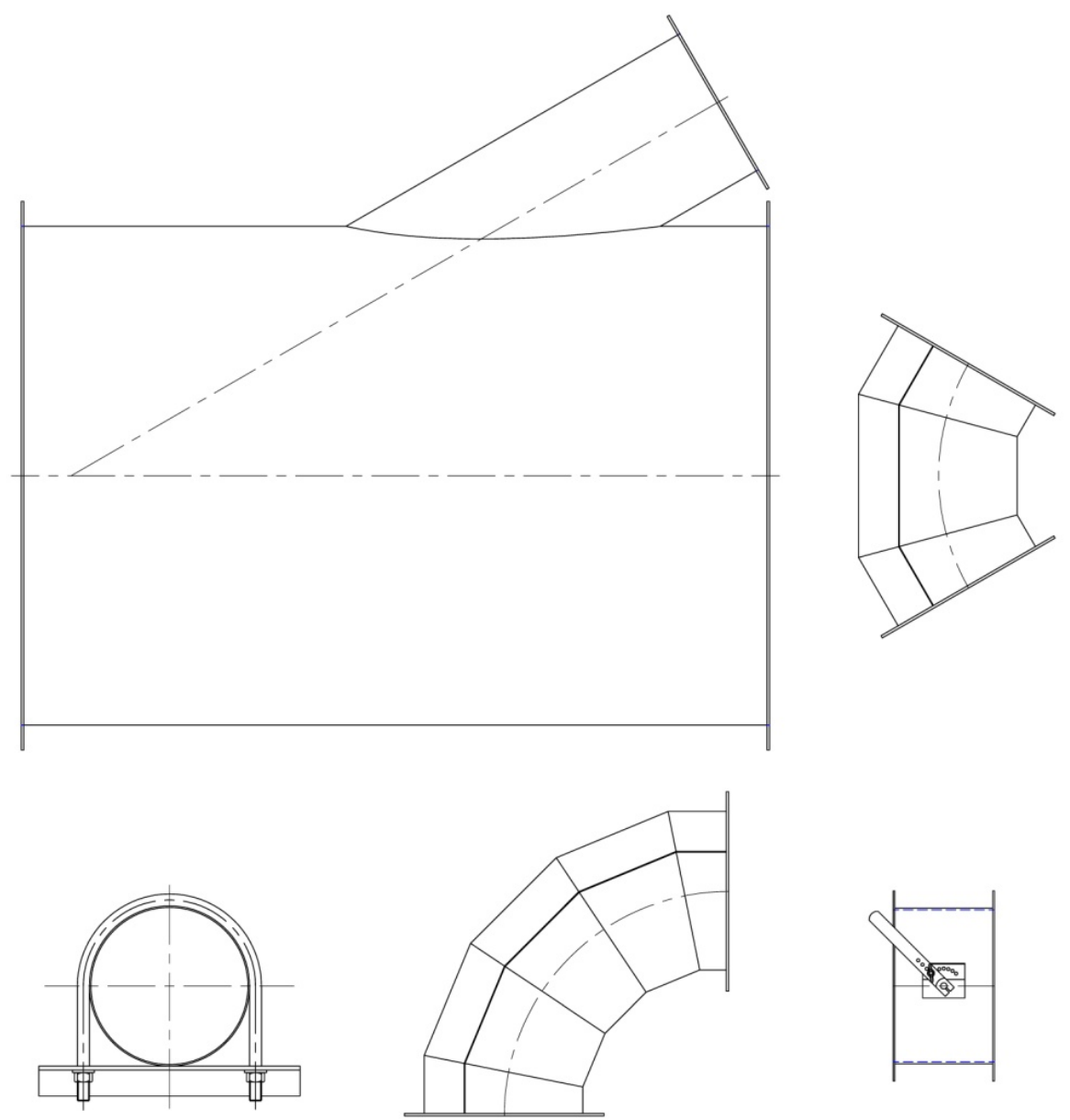

Figure 3 Elements of the pipeline for the secondary crusher dedusting

\section{CALCULATION}

Calculation is aimed to determination of pressure drop at dusty air flow through the pipeline section for secondary crusher dedusting and it is given according to [5]. Based on the calculation results, the pipeline is dimensioned and the pipe network is balanced.

1. Calculation the inner diameter of the pipeline is determined by the following formula: 


$$
d_{r}=\sqrt{\frac{4 \cdot Q}{3600 \cdot \pi \cdot v_{r}}}=0.326[\mathrm{~m}]
$$

Where:

$$
\begin{aligned}
Q & =6000\left[\frac{m^{3}}{h}\right]-\text { volumetric air flow } \\
v_{r} & =20\left[\frac{\mathrm{m}}{\mathrm{s}}\right] \text { - calculating air velocity }
\end{aligned}
$$

The standard outer pipeline diameter is adopted for ventilation piping installation $d_{s p}=0.315[\mathrm{~m}]$ which corresponds to the inner diameter of the pipeline of

$$
d=0.309[\mathrm{~m}]
$$

(for pipe wall thickness $\delta=3[\mathrm{~mm}]$ ), and the actual air velocity is calculated:

$$
v=\frac{4 \cdot Q}{3600 \cdot \pi \cdot d^{2}}=22.23\left[\frac{\mathrm{m}}{\mathrm{s}}\right]
$$

2. Friction loss coefficient is determined by the following formula:

\begin{tabular}{|c|c|}
\hline Conical convergent opening with wall at the end $\theta=0^{\circ}$ & 0.50 \\
\hline Damper, control, round $\theta=0^{\circ} \mathrm{D} / \mathrm{Do}=1$ & 0.19 \\
\hline Bend, 5 segments, round $\theta=90^{\circ} \mathrm{r} / \mathrm{D}=1.5$ pieces: 5 & 1.20 \\
\hline Bend, 3 segments, round $\theta=60^{\circ} \mathrm{r} / \mathrm{D}=1.5$ & 0.27 \\
\hline $\begin{array}{l}\text { Branch convergent } V \text { type, } 30^{\circ} \text {, round, } A_{2}=A_{1} \text {, } \\
\text { connection } A_{3} / A_{1}=0,1 ; Q_{3} / Q_{1}=0,1\end{array}$ & 0.24 \\
\hline & 2.40 \\
\hline
\end{tabular}

$$
\lambda=0.013+\frac{0.001}{d}=0.016
$$

3. The sum of point loss coefficients of the pipeline is determined according to [6] as follows:
4. Critical velocity is checked using the following formula:

$$
\begin{aligned}
v_{k r} & =0.3 \cdot \sqrt{c \cdot g \cdot d \cdot \frac{\rho_{\check{c}}}{\rho_{v}}}= \\
& =1.67\left[\frac{m}{s}\right]<22.23\left[\frac{m}{s}\right]
\end{aligned}
$$

Where:

$$
c=0.00333\left[\frac{k g_{\check{c}}}{k g_{v}}\right] \text { - solid particles con- }
$$
centration in the air

$$
\begin{aligned}
\rho_{\check{c}} & =3700\left[\frac{\mathrm{kg}}{\mathrm{m}^{3}}\right] \text { - solid particles density } \\
\rho_{v} & =1.2\left[\frac{\mathrm{kg}}{\mathrm{m}^{3}}\right] \text { - air density } \\
g & =9.81\left[\frac{\mathrm{m}}{\mathrm{s}^{2}}\right] \text {-gravitational acceleration }
\end{aligned}
$$

5. Pressure drop for clean air flow is determined by the following formula:

$$
\begin{aligned}
\Delta p_{v} & =\left(l \cdot \frac{\lambda}{d}+\Sigma \varsigma\right) \cdot \frac{\rho_{v} \cdot v^{2}}{2}= \\
& =1040.3[\mathrm{~Pa}]
\end{aligned}
$$

Where:

$l=21.1[\mathrm{~m}]-$ pipeline length

6. Pressure increase factor is:

$$
\Omega=0.02 \cdot \frac{v^{2}}{g \cdot \frac{d}{2} \cdot \cos \beta}=6.52
$$

Where:

$\beta=90\left[^{\circ}\right]$ - pipeline inclination angle

7. Pressure drop for dusty air flow is:

$\Delta p_{\check{c}}=\Delta p_{v} \cdot(1+k \cdot c)=1045.1[P a]$ Where:

$k=1.4$ za $\Omega>1$ - pressure increase coefficient 


\section{DISCUSSION OF CALCULATION}

As it can be seen, the calculation diameter of the pipeline is determined on the basis of the recommended air velocity for this type of installation, which amounts $v_{r}=20\left[\frac{\mathrm{m}}{\mathrm{s}}\right]$.

The standard value of the pipeline diameter for ventilation piping installation is adopted and on its basis the actual velocity is calculated which to a lesser extent deviates from the calculation velocity. Then the pressure drop is calculated in the pipeline due to the friction losses and point losses under assumption that the air is clean. The effect of dust presence on pressure drop at dusty air flow is taken into account through the pressure increase factor $\Omega$ and the pressure increase coefficient $\mathrm{k}$. The results obtained by calculation show that for selected operating parameters the effect of dust presence on pressure drop is negligible in relation to the pressure drop at clean air flow what may not always be the case.

In case of flow the heterogeneous mixture of fluid and solid particles such as dusty air flow, it is checked that the value of air velocity in the pipeline is above the critical rate at which deposition of dust particles in the pipeline takes place. As it can be seen from calculation, this requirement is met here.

The pipeline for secondary crusher dedusting represents only one branch of the installation for dust extraction from Old Flotation Crushing Facility which contains, as it was mentioned before, the total of ten suction places and the same number of flow circuits. Total pressure drop in flow circuit for the secondary crusher dedusting is compared to the pressure drop in the most unfavourable flow circuit and the remainder between these two values is determined.
This remainder is the value of pressure which has to be choked at round control damper and i amounts here $\Delta p=$ $262.6[\mathrm{~Pa}]$. By choking in such way at the all rest of the flow circuits (except the most unfavourable flow circuit, i.e. flow circuit with the highest pressure drop which represents the reference pressure loss), the aero dynamic balancing of the pipe network is done.

\section{CONCLUSION}

Dimensioning pipeline on the basis of the recommended speed is relatively simple method for design the pipeline installation. Certainly, it should be taken into account the all specifics of dusty air installation. Calculation of pressure drop in the dusty air flow deviates very little from calculation the pressure drop in the clean air flow, i.e. the effect of dust presence of dust is included by the appropriate corrective coefficient that depends on the air velocity, dust concentration and geometric parameters of pipeline.

\section{REFERENCES}

[1] Complementary Mining Project of Reconstruction the Facility for Smelter Slag Processing in the Flotation Plant Bor, Mining and Metallurgy Institute Bor, 2015 (in Serbian)

[2] Serbian Standard SRPS Z.B0.001: Maximum Allowable Concentrations of Toxic Gases, Vapours and Aerosols in the Atmosphere of Working Premises and Worksites, "Official Gazette SFRY“, No. 54/91 (in Serbian)

[3] Detail Mechanical Design for Dedusting the Slag Crushing Plant, 
Mining and Metallurgy Institute Bor; Section MECA; 2015 (in Serbian).

[4] Regulations on Emission the Limit Values, Methods and Time Limits for Measuring and Data Recording, "Official Gazette of the Republic of Serbia", Nos. 30/97 and 35/97 (in Serbian)
[5] M. Bogner, M. Stojanović, L. Livo Purification and Filtration of Gases and Liquids, ETA, Belgrade, 2006 (in Serbian)

[6] B. Todorović, M. Milinković, Air Distribution in the air Conditioning Systems, SMEITS, Belgrade, 1991 (in Serbian) 